ISSN 1997-5902

\title{
Distribution spatiale intra-urbaine des particules fines: monitoring par l'Aimantation Rémanente Isotherme à Saturation des feuilles (SIRM) en milieu tropical urbain (Côte d'Ivoire).
}

\author{
N'GOURAN Kobenan Pierre', BARIMA Yao Sadaiou Sabas², ANGAMAN Djédoux Maxime ${ }^{3}$, KOFFI \\ N'guessan Achille ${ }^{3}$, TRA BI Zamblé Fidèle 3 , DA Kouhété Philippe ${ }^{1}$ DONGUI Bini Kouamé ${ }^{2}$ \\ 1 Unité de Formation et de Recherche en Biosciences, Université Félix Houphouët Boigny, 01 BP V 34 Abidjan, Côte \\ d'Ivoire. \\ 2Unité de Formation et de Recherche en Environnement, Université Jean Lorougnon Guédé, BP 150 Daloa, Côte d'Ivoire. \\ 'Unité de Formation et de Recherche en Agroforesterie, Université Jean Lorougnon Guédé, BP 150 Daloa, Côte d'Ivoire. \\ Auteur correspondant : N'GOURAN Kobenan Pierre ; Email : ngoranpierre@yahoo.fr
}

Original submitted in on $8^{\text {th }}$ July 2014. Published online at www.m.elewa.org on 30 th September 2014. http://dx.doi.org/10.4314/jab.v81i1.7

\section{RESUME}

Objectif: Le présent travail vise à déterminer les teneurs en particules fines à partir de l'Aimantation Rémanente Isotherme (SIRM) des feuilles de plantes tropicales dans le District d'Abidjan.

Méthodologie et résultats : Des échantillons de feuilles de plantes de Amaranthus spinosus, Panicum maximum, Ficus benjamina et Terminalia catappa ont été prélevés dans différents types d'utilisation du sol que sont : les axes routiers ; les zones industrielles; les zones résidentielles et les parcs et jardins. Les valeurs les plus élevées de SIRM $\left(22,33 \times 10^{-5} \mathrm{~A}\right.$ et $\left.7,36 \times 10^{-5} \mathrm{~A}\right)$ ont été enregistrées avec les feuilles de Amaranthus spinosus respectivement en zones industrielles et au niveau des axes routiers. Au contraire, dans les zones résidentielles et les parcs et jardins, les SIRM des feuilles sont très faibles avec des valeurs variant entre 2,62 $x 10^{-5} \mathrm{~A}$ et $0,33 \times 10^{-5} \mathrm{~A}$ au cours des différentes saisons. Les résultats ont révélé également un gradient croissant des SIRM des feuilles à mesure que l'on se rapproche des routes et un gradient décroissant avec la hauteur d'échantillonnage. En outre, l'accumulation des particules fines est faible pendant un mois pluvieux par rapport à un mois sec.

Conclusion : Les résultats de cette étude ont permis de ranger les types d'utilisation du sol étudiés en deux catégories selon la qualité de l'air. Ainsi les zones industrielles et les abords des principaux axes routiers sont des zones de mauvaise qualité d'habitat car plus pollués que les zones résidentielles et les parcs et jardins qui sont des zones de bonne qualité d'habitat. Aussi, cette étude a révélé que les principales sources de la pollution de l'environnement en milieu urbain sont d'une part les émissions des gaz d'échappement des véhicules et d'autre part les cheminées des usines. En somme, le SIRM des feuilles des plantes peut être utilisé comme un bio-indicateur de la qualité de l'air dans un environnement urbain en milieu tropical.

Mots clés : biomonitoring, particules fines, Aimantation Rémanente Isotherme à Saturation (SIRM), qualité de l'habitat, plantes tropicales. 
Intra-urban spatial distribution of particulate matter: monitoring by leaf Saturation Isothermal Remanent Magnetization (SIRM) in tropical urban areas (Côte d'Ivoire).

\section{Abstract:}

Objective: The aim of this study was to determine particulate matter concentration from the Saturation Isothermal Remanent Magnetization (SIRM) of tropical leaves in the District of Abidjan.

Methodology and Results : Leaves sampling of Amaranthus spinosus, Panicum maximum, Ficus benjamina and Terminalia catappa were taken from different land use types, i.e.: roads, industrial areas, residential areas and parks and gardens. The highest leaf SIRM $\left(22.33 \times 10^{-5} \mathrm{~A}\right.$ and $\left.7.36 \times 10^{-5} \mathrm{~A}\right)$, were recorded with Amaranthus spinosus respectively in industrial areas and at roads. Instead, in residential areas and parks and gardens, leaf SIRM varies between $2.62 \times 10^{-5} \mathrm{~A}$ and $0.33 \times 10^{-5} \mathrm{~A}$ during different seasons. Leaf SIRM showed an increasing gradient with distance to the roads and declining gradient with sampling height. Furthermore, the accumulation of fine particles is low for a rainy months compared to dry months.

Conclusion: This study has allowed to classify the land use types in to two categories according to air quality. Industrial areas and roads areas are poor air quality habitat but residential areas and parks and gardens areas are good air quality habitat. Also, this study revealed that the main sources of environmental pollution in urban areas are emissions from motors vehicles and industries chimneys. This study has showed that leaf SIRM of tropical plants leaves can be used as bioindicators of air quality in urban environments in the tropics.

Keywords : biomonitoring, particulate matter, Saturation Isothermal Remanent Magnetization, habitat quality, tropical plants.

\section{INTRODUCTION}

L'industrialisation et l'urbanisation galopante ces dernières décennies sont à l'origine d'une concentration de plus en plus élevée de particules polluantes dans la plupart des grandes villes du monde et en particulier dans les pays en voie de développement. Ainsi, la qualité de l'air est susceptible d'être la question de l'environnement la plus préoccupante dans l'avenir (Beaumont et al., 1999). Les effets néfastes de la pollution atmosphérique sur la santé humaine sont connus (Emberson et al., 2001). Les dommages des particules fines (PM) sur la santé des populations et les changements microclimatiques au sein des villes sont importants. Le District d'Abidjan, la capitale économique de la Côte d'Ivoire, avec une population estimée à plus de quatre millions d'habitant (INS, 1998) possède un parc automobile constitué à majorité de voitures d'occasion. La ville renferme aussi l'essentiel des usines et ports de Côte d'Ivoire. Tous ces facteurs sont susceptibles d'augmenter la pollution de l'air notamment en PM dans cet environnement tropical. Les PM, d'origine anthrophiques et de tailles variables sont composés entre autres de métaux lourds tels que le Zinc, le Cadmium et le Chrome (Georgeaud et al., 1997).
Les effets néfastes des PM sur la santé de l'Homme sont reconnus à cause de leur facilité à être inhalée et à se fixer dans les poumons (Prajapati et al., 2005) provoquant de nombreuses maladies respiratoires et cardiovasculaires (Moreno et al., 2003). L'OMS (2003) estime à plus de 1,4\%, le nombre de décès dans le monde dû aux PM. En Côte d'lvoire, les données sur la pollution atmosphérique et les effets des particules fines sur la population sont peu disponibles. Les appareils de détection de particules pourraient être utilisés à cette fin mais leur coût élevé (Kardel et al., 2012), constitue une entrave pour les économies faibles des villes du Sud (Barima et al. 2014). En plus, ces appareils sont sensibles seulement à une gamme de polluants, les rendant inefficaces pour évaluer la répartition spatiale de la pollution de l'environnement (Kardel et al., 2012).

Une méthode alternative serait d'employer des bioindicateurs passifs comme les plantes. En général, les plantes sont plus sensibles aux polluants que les humains et les animaux (Nali \& Lorenzini, 2007). En particulier, les feuilles, qui occupent une grande place dans l'interaction entre la plante et l'atmosphère sont continuellement 
exposées à la pollution de l'air. Ces feuilles peuvent ainsi intercepter une quantité variable de polluants (Bealey et al., 2007). Aussi, le biomonitoring des PM via l'Aimantation Rémanente Isotherme à Saturation des feuilles (SIRM) des plantes est connu pour être une technique rapide, non destructive et peu coûteuse (Kardel et al., 2011; Barima et al., 2014). L'objectif de cette étude est de déterminer les teneurs en particules fines de deux herbacées (Amaranthus spinosus et Panicum maximum) et deux arborées (Ficus benjamina et Terminalia catappa) dans le District d'Abidjan en Côte d'Ivoire à

\section{MATERIEL ET METHODES}

Localisation du site: Cette étude a été effectuée dans le District d'Abidjan $\left(5^{\circ} 00^{\prime}-5^{\circ} 30^{\prime} \mathrm{N}\right.$ et $\left.3^{\circ} 50^{\prime}-4^{\circ} 10^{\prime} \mathrm{W}\right)$ avec une superficie de $2119 \mathrm{~km}^{2}$ soit $0,6 \%$ du territoire total de la Côte d'Ivoire. Ce District jouit d'un climat de type subéquatorial, chaud et humide avec une grande saison des pluies (mai-juin-juillet), une petite saison des pluies (septembre-novembre) et deux saisons sèches. La pluviométrie annuelle est d'environ1 $800 \mathrm{~mm}$ (Ahoussi et al., 2013). Dans le District d'Abidjan, les précipitations mensuelles varient entre $26 \mathrm{~mm}$ et $610 \mathrm{~mm}$ et le degré d'hygrométrie y atteint $80 \%$. La température dans cette partie de la Côte d'Ivoire varie entre 25 et $28^{\circ} \mathrm{C}$ au cours de l'année. La campagne d'échantillonnage a été effectuée pendant les mois de mars et juin. Avec 43,18 $\mathrm{mm}$ de pluie et une température de $28,30^{\circ} \mathrm{C}$, le mois de mars est le mois le plus sec et le plus chaud de cette campagne tandis le mois de juin est le plus pluvieux avec une hauteur de pluie de $375,91 \mathrm{~mm}$ et une température de $26^{\circ} \mathrm{C}$ (www.tutiempo.net/clima/Abidjan/).

Plan d'échantillonnage : Dans le cadre de cette étude, le District d'Abidjan a été subdivisé en quatre (04) types d'utilisation du sol suivants : les zones industrielles (ZI) ; les zones résidentielles (ZR) ; les parcs et jardins (PJ) et les principaux axes routiers (AR). Le type AR regroupe cinq (05) axes routiers à densité de trafic relativement intense et variable. Dans une étude précédente, le partir de l'aimantation rémanente isotherme des feuilles. Les quatre différents objectifs spécifiques suivants permettront d'atteindre cet objectif : (i) déterminer la distribution des SIRM des feuilles dans les différents types d'utilisation du sol dans le District d'Abidjan, (ii) déterminer la répartition des SIRM des feuilles suivant la distance à la route, (iii) déterminer la répartition en hauteur des SIRM des feuilles des arbres situés aux abords des axes routiers, (iv) déterminer l'impact des saisons sur la teneur des SIRM des feuilles.

nombre de voitures passant sur ces principaux axes routiers dans un laps de temps a été rélevé pendant les périodes de grands trafics et extrapolé à l'heure après 10 répétitions. Le type AR comprend donc les routes suivantes : l'autoroute du Nord (4128 véhicules/heure), le boulevard Lagunaire ( 8280 véhicule/heure), le boulevard de la Paix (3228 véhicules/heure), le boulevard Latrille (2796 véhicules/heure) et la route du Zoo (2124 véhicules/heure). Le type ZI regroupe les deux (02) plus grandes zones industrielles de la ville d'Abidjan que sont les zones industrielles de Yopougon et de Vridi. Les PJ renferment le Centre National de Floristique et le Jardin Botanique de Bingerville. Enfin, le type ZR renferme trois (03) quartiers résidentiels (Riviera, Abobo et Yopougon) (Fig. 1). Sur chaque axe routier, deux sites d'échantillonnages distants d'au moins $2 \mathrm{~km}$ ont été choisis. Sur chaque site, trois prélèvements ont été effectués sur chaque herbacée en s'éloignant du point de prélèvement le plus proche de la route. Pour les arborées, les feuilles ont été prélevées à trois niveaux de hauteur : inférieur à $1 \mathrm{~m}$, entre 1 et $2 \mathrm{~m}$ et au delà de $2 \mathrm{~m}$. Dans chacun des autres types, trois sites d'échantillonnage ont été choisis. Trois points de prélèvement des feuilles sont identifiés dans chacun de ces sites. 


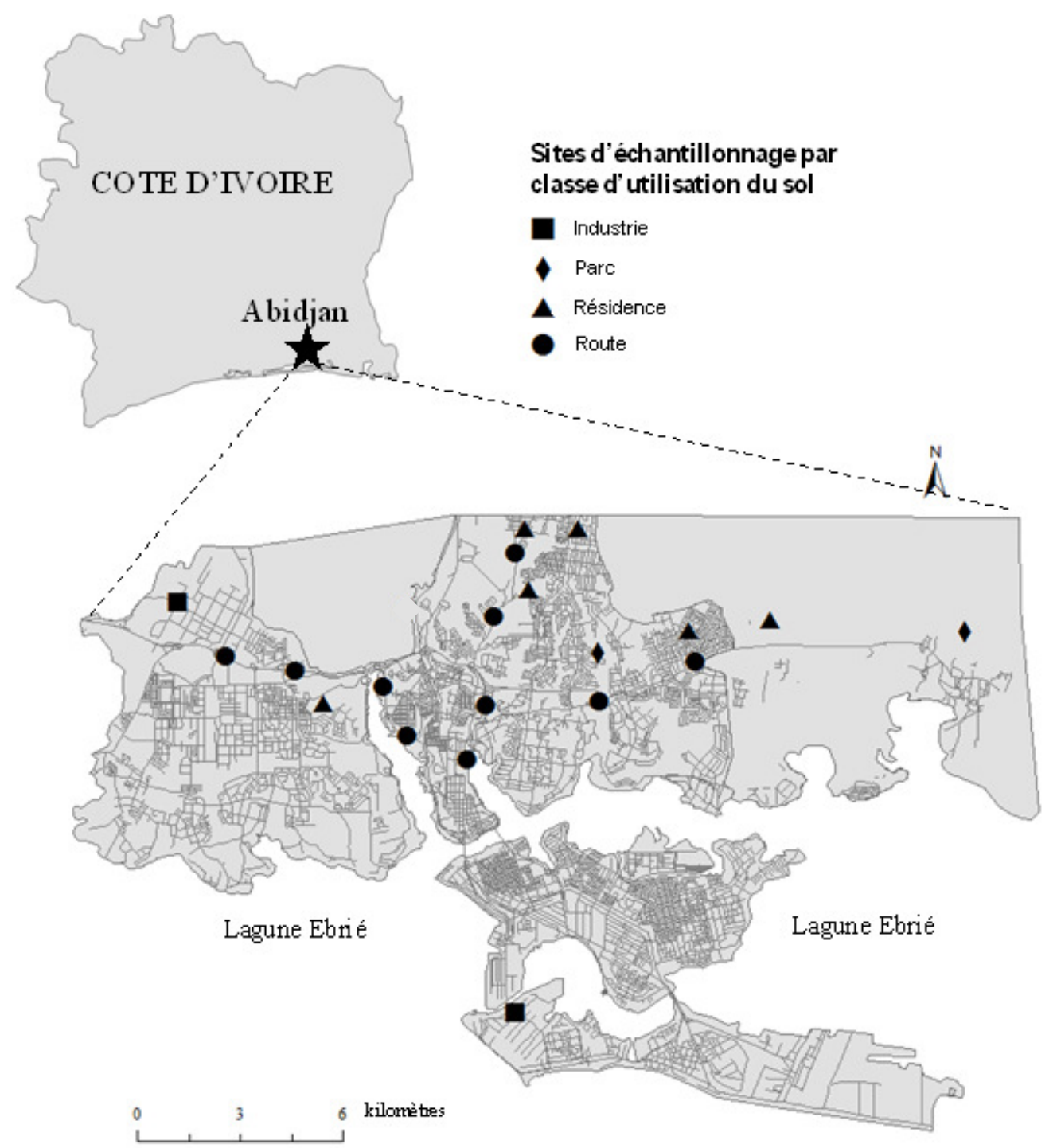

Figure 1 : Localisation du District d'Abidjan en Côte d'Ivoire et des différentes zones d'échantillonnage dans les quatre (04) types d'utilisation du sol.

Sélection et description des espèces : Un inventaire botanique itinérant a été effectué dans les différents types d'utilisation du sol listés plus haut. Ces inventaires ont été réalisés en notant la présence ou l'absence d'une espèce dans le milieu. De plus, les plantes sélectionnées devraient avoir des feuilles suffisamment grandes afin de faciliter la réalisation de différents tests. Ainsi, les espèces microphylles de même que les espèces à feuilles à aiguilles n'ont pas été retenues. Les deux herbacées et les deux arborées les plus répandues, les mieux représentées, les plus abondantes dans les différents types d'utilisation du sol ont été retenues.
Prélèvement des feuilles et détermination des paramètres environnementaux : Trois feuilles érigées, matures et non endommagées ont été prélevées sur chaque plante à chaque point d'échantillonnage. Sur chaque axe routier, les feuilles échantillonnées étaient orientées du côté de la route. Les feuilles en contact avec le sol ont été évitées afin de minimiser la contamination directe des feuilles par la terre. La géolocalisation de chaque site a été précisée grâce à un GPS (Global Positioning System).

Mesures des caractéristiques morphologiques et conditionnement des feuilles : Les feuilles ont été méticuleusement scannées le jour des prélèvements en 
évitant les contaminations par les objets métalliques. Les images scannées ont servi à déterminer la surface des feuilles grâce au logiciel Image J. La détermination de la surface des feuilles permettra d'estimer la teneur en particules fines par unité de surface. Chaque échantillon de feuilles a été ensuite mis à sécher à température ambiante. Après séchage, chaque lot de feuilles a été enroulé fermement dans un film alimentaire, puis préssé dans un tube plastique de $10 \mathrm{~cm}^{3}$.

Détermination de SIRM : La détermination des SIRM des feuilles a été réalisée suivant un protocole mis en place par Matzka et Maher (1999) et utilisée par plusieurs auteurs dont Prajapati et al. (2005), Mitchell et al. (2010), Kardel et al. (2012), Barima et al. (2014). L'échantillon préalablement placé dans le tube plastique de $10 \mathrm{~cm}^{3}$ est d'abord magnétisé avec un champ magnétique de 1 Tesla grâce au Molspin pulse Magnetiser (Molspin Ltd, UK). Ensuite la Magnétisation Rémanente Isotherme (IRM) est mesurée avec un magnétomètre (Molspin Minispin ${ }^{\circledR}$ ). L'appareil est calibré avant et après dix

\section{RESULTATS}

Espèces retenues : Les deux herbacées et les deux arborées les plus largement répandues, les mieux représentées et les plus abondantes dans les différents types d'utilisation du sol retenues à la suite de l'inventaire botanique sont : Amaranthus spinosus L. (Amaranthaceae), Panicum maximum Jacq. (Poaceae), Ficus benjamina L. (Moraceae) et Terminalia catappa L. (Combretaceae). A. spinosus est une herbacée cosmopolite, annuelle, érigée, monoïque, atteignant 100 à $130 \mathrm{~cm}$ de haut et fortement ramifiée. Ses feuilles simples sont alternes avec absence de stipules. Son pétiole est aussi long que le limbe de la feuille qui est ovale-lancéolé à rhomboïde, de 3,5-11 cm x 1-4,5 cm, aigu et souvent légèrement décurrent à la base et brièvement mucroné à l'apex. Ses nervures sont légèrement pubescentes à l'état jeune. P. maximum est une herbe vivace, érigée, glabre, abondamment ramifiée. Les feuilles, ovales ou elliptiques-lancéolées ont une dimension de 3-8 $\mathrm{cm} \times 2-4 \mathrm{~cm}$ avec un pétiole de $4 \mathrm{~cm}$ de long. Cette herbe est communément utilisée comme plante fourragère. F. benjamina est un arbre tropical pouvant atteindre $30 \mathrm{~m}$ de haut dans des conditions naturelles. Ses feuilles sont coriaces, pétiolées avec une forme ovale-acuminée de $6-13 \mathrm{~cm}$ de long. Cette espèce est utilisée comme plante ornementale et atteint alors rarement plus de $3 \mathrm{~m}$. T. catappa est un arbre à feuilles mesures comme décrit par Matzka et Maher (1999). Chaque IRM retenu est une moyenne de 2 mesures d'IRM du même échantillon. Les SIRM des feuilles sont obtenus en ramenant l'IRM à la surface des feuilles contenues dans le tube de $10 \mathrm{~cm}^{3}$. La détermination des IRM a été réalisée au Laboratory of Environmental and Urban Ecology, Department of Bioscience Engineering, University of Antwerp (Belgique).

Analyses statistiques : La normalité des données des SIRM des feuilles prélévées en mars et en juin a été évaluée par le test de Kolmogorove Smirnov. La comparaison des SIRM des feuilles par type d'utilisation du sol a été réalisée à partir d'une ANOVA (Analysis of variance) à un facteur (test de Tukey-HSD). Des tests $t$ de Student ont été effectués pour comparer les SIRM des feuilles de la même espèce au cours des deux saisons (sèche et pluvieuse) d'échantillonnage. Différents modèles de régressions ont servi à trouver les liens entre les SIRM des feuilles et les distances de prélevements des feuilles par rapport à la route.

caduques à croissance rapide de 9 à $25 \mathrm{~m}$ de haut et de 60 à $70 \mathrm{~cm}$ de diamètre. Les feuilles groupées à l'extrémité des branches sont portées par un gros pétiole, tomenteux puis glabre, de 5-17 mm. Le limbe est oboval, à base cunéiforme, à apex rond, de $8-36 \mathrm{~cm} \times 6-24 \mathrm{~cm}$. Distribution des SIRM des feuilles dans les différents types d'utilisation du sol : Les différentes valeurs de SIRM des feuilles enregistrées au cours de cette campagne d'échantillonnage varient au sein du même type d'utilisation du sol ainsi qu'entre les différents types (Tableau 1). Au niveau des axes routiers (AR), le SIRM des feuilles le plus élevé a été enregistré avec $F$. benjamina $\left(12,86 \times 10^{-5} \mathrm{~A}\right)$ et le qu'entre les différents types plus faible SIRM avec $P$. maximum $\left(4,90 \times 10^{-5} \mathrm{~A}\right)$ (Tableau 1). En ce qui concerne les zones industrielles (ZI), le SIRM des feuilles de $A$. spinosus qui s'élève à $22,23 \times 10^{-5} \mathrm{~A}$ est la plus grande valeur de SIRM enregistrée au cours de cette campagne. En ZI comme dans les parcs et jardins (PJ), les SIRM des feuilles donnent chez toutes les 4 espèces des valeurs assez faibles. Cependant, $F$. benjamina avec des SIRM des feuilles respectifs de $2,57 \times 10^{-5} \mathrm{~A}(\mathrm{ZR})$ et de $1,76 \times 10^{-5} \mathrm{~A}$ (PJ) a les plus grandes valeurs dans ces deux types (Tableau 1). 


\section{N'gouran et al. J. Appl. Biosci. 2014. Distribution spatiale intra-urbaine des particules fines : Monitoring}

par SIRM des feuilles en milieu urbain, Cote d'Ivoire

Tableau 1: Moyenne des SIRM des feuilles ( \pm écart-type) de Amaranthus spinosus, Panicum maximum, Ficus benjamina et Terminalia catappa échantillonnés au cours de la saison sèche (mars) et la saison pluvieuse (juin) dans les différents types d'utilisation du sol (TUS).

\begin{tabular}{lllllll}
\hline TUS & Espèces & SIRM $\left(\mathbf{x} \mathbf{1 0}^{-\mathbf{5}} \mathbf{A}\right)$ & & $\mathbf{t}$ & $\mathbf{p}$ value & $\mathbf{n}$ \\
& & Mars & Juin & & & \\
\hline \multirow{4}{*}{ AR } & Amaranthus spinosus & $10,37 \pm 9,55$ & $7,36 \pm 4,98$ & 1,702 & 0,100 & 26 \\
& Panicum maximum & $4,90 \pm 2,58$ & $2,79 \pm 1,77$ & 3,551 & $\mathbf{0 , 0 0 1}$ & 26 \\
& Ficus benjamina & $12,86 \pm 10,27$ & $5,41 \pm 3,79$ & 4,953 & $\mathbf{0 , 0 0 0}$ & 45 \\
& Terminalia catappa & $9,58 \pm 10,13$ & $5,06 \pm 4,66$ & 1,305 & 0,220 & 12 \\
\hline \multirow{4}{*}{ ZI } & Amaranthus spinosus & $22,23 \pm 17,61$ & $3,87 \pm 3,90$ & 3,405 & $\mathbf{0 , 0 0 5}$ & 12 \\
& Panicum maximum & $8,63 \pm 4,96$ & $1,30 \pm 1,11$ & 5,586 & $\mathbf{0 , 0 0 0}$ & 12 \\
& Ficus benjamina & $7,63 \pm 6,46$ & $6,26 \pm 3,05$ & 0,868 & 0,397 & 18 \\
& Terminalia catappa & $12,98 \pm 7,88$ & $2,79 \pm 1,57$ & 3,197 & $\mathbf{0 , 0 2 4}$ & 6 \\
\hline \multirow{4}{*}{ ZR } & Amaranthus spinosus & $1,83 \pm 1,07$ & $1,51 \pm 1,07$ & 1,18 & 0,253 & 18 \\
& Panicum maximum & $2,46 \pm 1,41$ & $0,33 \pm 0,10$ & 6,558 & $\mathbf{0 , 0 0 0}$ & 18 \\
& Ficus benjamina & $2,57 \pm 0,81$ & $1,03 \pm 0,49$ & 9,012 & $\mathbf{0 , 0 0 0}$ & 27 \\
\hline \multirow{2}{*}{ PJ } & Terminalia catappa & $2,62 \pm 2,20$ & $1,08 \pm 0,81$ & 2,464 & $\mathbf{0 , 0 3 1}$ & 12 \\
& Panicum maximum & $1,00 \pm 0,16$ & $0,41 \pm 0,10$ & 7,619 & $\mathbf{0 , 0 0 0}$ & 12 \\
\hline AR & Ficus benjamina & $1,76 \pm 0,77$ & $1,23 \pm 0,49$ & 2,359 & $\mathbf{0 , 0 3 0}$ & 18 \\
\hline
\end{tabular}

$\mathrm{AR}=$ principaux axes routiers $; \mathrm{ZI}=$ Zones industrielles $; \mathrm{ZR}=$ Zones résidentielles et $\mathrm{PJ}=$ parcs et jardins. $\mathrm{t}=$ test de Student. Les différences significatives $(p \leq 0,05)$ sont présentées en gras. $n=$ nombre d'échantillons.

Distribution horizontale des SIRM des feuilles des herbacées : Les différentes mesures de SIRM des feuilles de $A$. spinosus et $P$. maximum obtenues au cours de cette campagne ont révélé que leur valeur diminue en général au fur et à mesure qu'on s'éloigne de la route (Fig. 2). Cependant, pendant la saison des pluies, la plus faible valeur de SIRM des feuilles de $A$. spinosus (4,58 $x 10^{-5} \mathrm{~A}$ ) a été mesurée entre 2 et $3,5 \mathrm{~m}$ et non au-delà de
3,5 m. De même, la plus faible valeur de SIRM des feuilles de $P$. maximum pendant la saison sèche a été enregistrée juste au bord de la route c'est-à-dire entre 0 et $2 \mathrm{~m}$ (Tableau 2). En outre, les analyses statistiques ont montré seulement une différence significative entre les SIRM de $A$. spinosus mesurés dans les intervalles 0 à 2 $m$ et 2 à 3,5 m en juin (Tableau 2 et Fig. 2).

Tableau 2 : SIRM des feuilles ( \pm écart-type) $\left(\times 10^{-5} \mathrm{~A}\right)$ de Amaranthus spinosus et Panicum maximum prélevés en saison des pluies (mars) et en saison sèche (juin) suivant la distance à la route.

\begin{tabular}{lllll}
\hline & \multicolumn{4}{l}{ Distance par rapport à la route $(\mathbf{m})$} \\
\hline Espèces & Mois & $\mathbf{0 - 2}$ & $\mathbf{2 - 3 , 5}$ & $\mathbf{2 3 , 5}$ \\
\hline \multirow{2}{*}{ Amaranthus spinosus } & mars & $11,76 \pm 9,50$ & $8,71 \pm 5,3^{\mathrm{A}}$ & $6,31 \pm 6,77$ \\
& juin & $8,42 \pm 3,81^{\mathrm{a}}$ & $4,58 \pm 1,56^{\mathrm{b}}$ & $5,67 \pm 1^{\mathrm{ab}}$ \\
\hline \multirow{2}{*}{ Panicum maximum } & mars & $4,38 \pm 1,84^{\mathrm{B}}$ & $4,97 \pm 1,42^{\mathrm{B}}$ & $4,62 \pm 3,28$ \\
& juin & $2,64 \pm 1,17^{\mathrm{A}}$ & $2,35 \pm 1,21^{\mathrm{A}}$ & $1,96 \pm 1,19$ \\
\hline
\end{tabular}

Les lettres en minuscule ( $a$ et $b$ ) indiquent une différence significative entre les SIRM des feuilles de la même plante dans les différents intervalles tandis que les lettres en majuscule ( $A$ et $B$ ) indiquent une différence significative entre les SIRM des feuilles pendant les 2 saisons dans le même intervalle $(p \leq 0,05)$. 


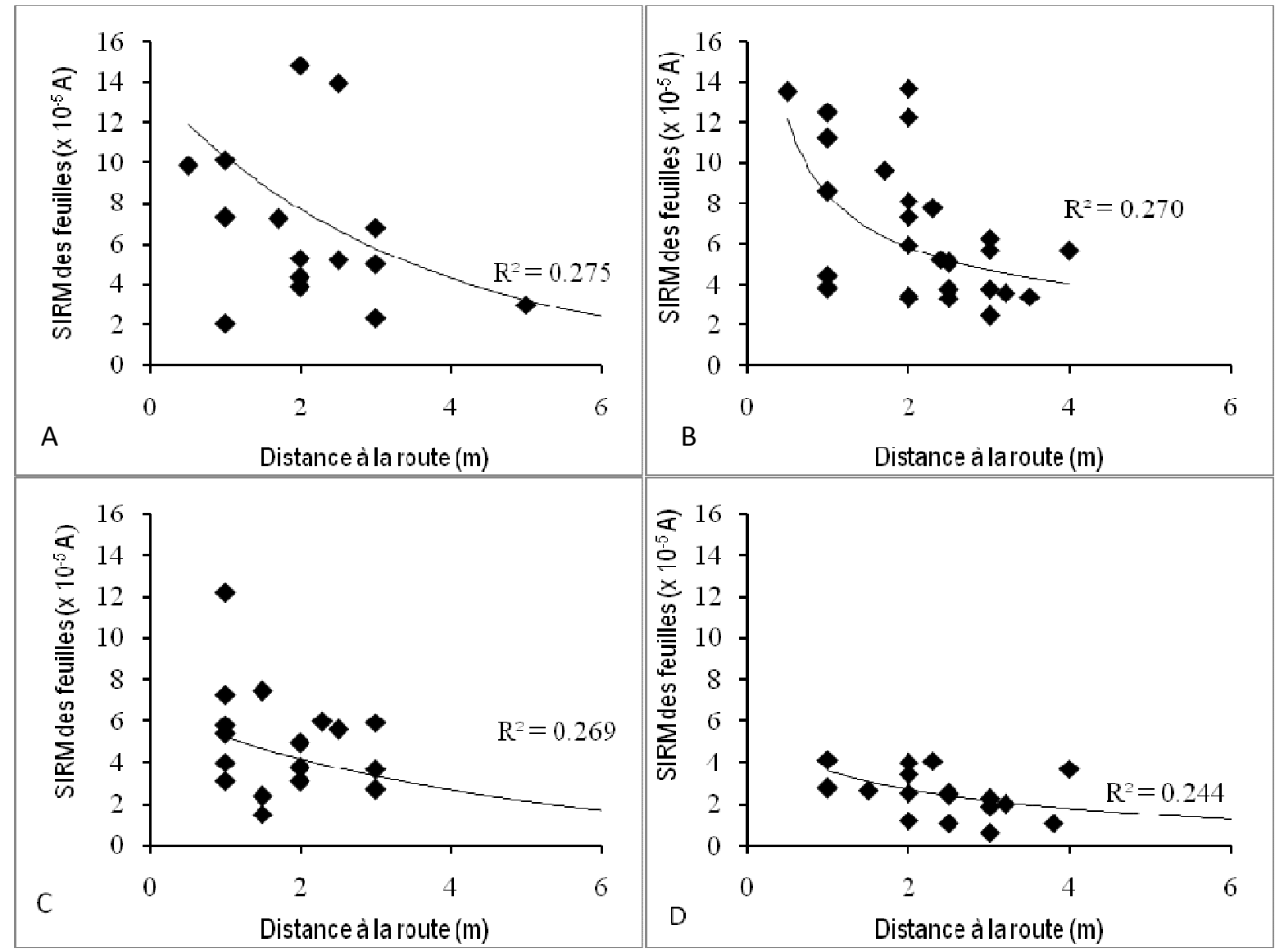

Figure 2 : Variation du SIRM des feuilles $\left(\times 10^{-5} \mathrm{~A}\right)$ de Amaranthus spinosus et Panicum maximum par rapport à la distance à la route. $A$ et $B$ représentent les échantillons respectivement de mars et juin de Amaranthus spinosus. $C$ et $D$ représentent les échantillons respectivement de mars et juin de Panicum maximum.

Distribution verticale des SIRM des feuilles des espèces arborées : La valeur des SIRM des feuilles de $F$. benjamina et $T$. catappa dépend de la hauteur de prélèvement des feuilles (Fig. 3). En mars, les SIRM des feuilles de F. benjamina diminuent avec la hauteur. Ainsi, la plus grande valeur de SIRM de cette espèce est obtenue entre 0 et $1 \mathrm{~m}$ tandis que la plus faible est enregistrée au-delà de $2 \mathrm{~m}$. Cependant, pour les SIRM des feuilles de $T$. catappa, la plus grande valeur est plutôt enregistrée entre 1 et $2 \mathrm{~m}$ et la plus petite valeur entre 0 et 1 mètre. En juin également, les valeurs de SIRM des feuilles des deux arborées sont contrastées selon la hauteur de prélèvement des feuilles. En effet, le SIRM des feuilles de $F$. benjamina le plus élevé est enregistré dans l'intervalle 0 à $1 \mathrm{~m}$ avec une valeur de $7,01 \times 10^{-5} \mathrm{~A}$ tandis que le faible SIRM a été enregistré entre 1 et $2 \mathrm{~m}$ (Fig. 3). Pour T. catappa, les feuilles échantillonnées en juin montrent un gradient décroissant en ce qui concerne les valeurs de SIRM avec une augmentation de la hauteur de prélèvement (Fig. 3). On remarque qu'en général, les valeurs de SIRM des feuilles les plus élevées sont obtenues entre 0 et $1 \mathrm{~m}$ et les plus faibles sont audelà de $2 \mathrm{~m}$. 


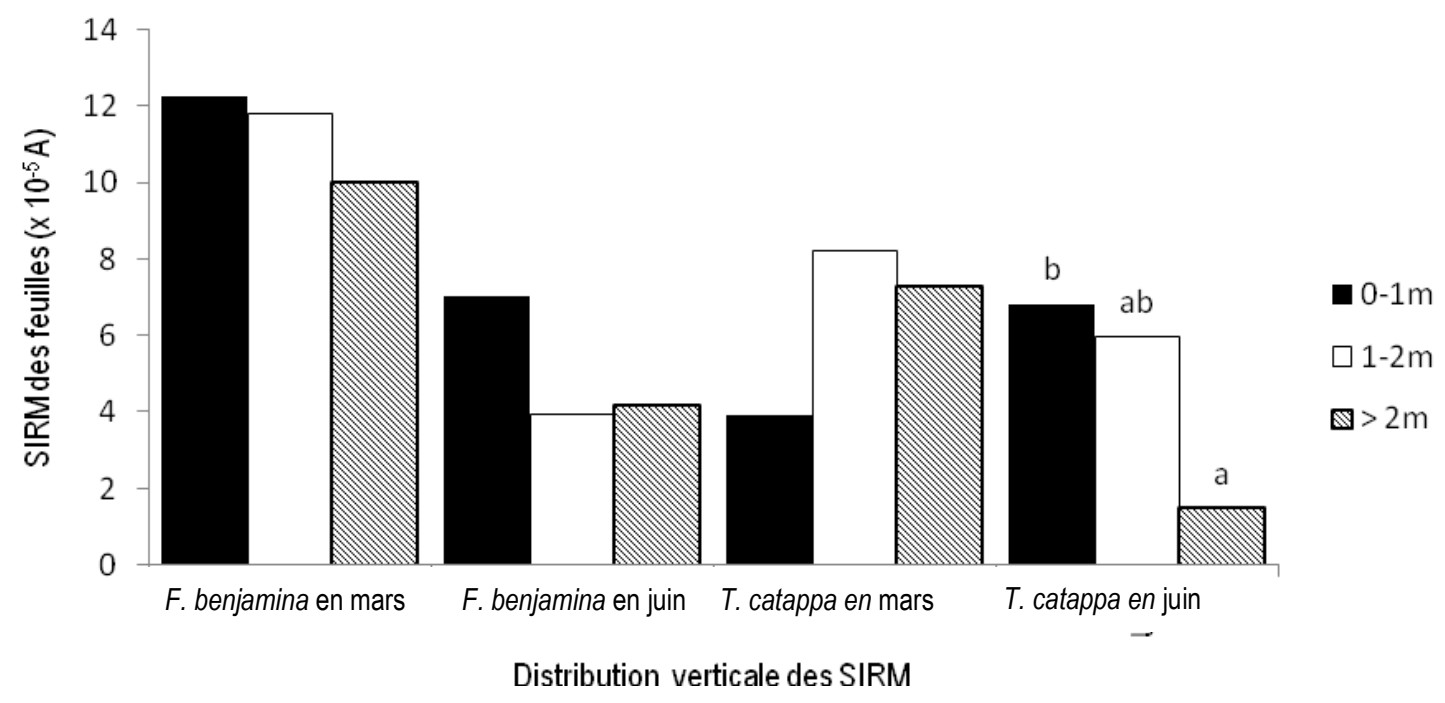

Figure 3 : Répartition des SIRM des feuilles de Ficus benjamina et Terminalia catappa en fonction de leurs hauteurs de prélèvement en mars (saison sèche) et juin (saison des pluies). Les lettres indiquent les différences significatives entre les SIRM des feuilles prélevées à différentes hauteurs à $p \leq 0,05$.

Effet des saisons sur les SIRM des feuilles : Les SIRM des feuilles des différentes espèces au cours de la saison sèche (mars) sont plus élevés que les SIRM des feuilles obtenus pendant la saison des pluies (juin) (Tableau 1). Les SIRM des feuilles de $A$. spinosus avec une valeur de $22,23 \times 10^{-5} \mathrm{~A}$ en sèche saison sèche chute à $3,87 \times 10^{-5} \mathrm{~A}$ en saison des pluies soit une perte de $82,59 \%$. Le SIRM le plus élevé $\left(8,63 \times 10^{-5} \mathrm{~A}\right)$ de $P$. maximum enregistré au

\section{DISCUSSIONS}

Distribution des SIRM des feuilles dans les types d'utilisation du sol : Les résultats de cette étude ont montré que les SIRM des feuilles sont inégalement répartis dans les différents types d'utilisation du sol dans notre zone d'étude (Tableau 1). Les SIRM des feuilles les plus élévés sont enregistrés dans les AR et $\mathrm{Zl}$ contrairement aux ZR et PJ qui ont des SIRM des feuilles faibles. Ainsi, selon la concentration des particules fines accumulées par les feuilles des différentes espèces de $A$. spinosus, $P$. maximum, $F$. benjamina et $T$. catappa, le District d'Abidjan peut être divisé en deux sous zones selon la qualité de l'air. Du fait de la relative faible teneur en SIRM des feuilles des plantes qui y sont présentes, les parcs et jardins et les zones résidentielles peuvent être considérés comme des zones à bonne qualité d'air alors que les types d'utilisation du sol à mauvaise qualité d'air sont constitués des principaux axes routiers et des zones industrielles. Ces résultats concordent avec les travaux de Kardel et al. (2012) qui ont montré que la cours de cette campagne en mars chute à $1,30 \times 10^{-5} \mathrm{~A}$ en juin soit une baisse de $84,93 \%$ du taux de particules fines. La diminution est de $57,93 \%$ et $78,50 \%$ entre la saison sèche et la saison pluvieuse pour $F$. Benjamina et $T$. catappa respectivement. Ces proportions obtenus sont en accord avec les analyses statistiques qui montrent l'existence d'une différence significative entre les SIRM des feuilles de mars et celles de juin (Tableau 1).

concentration atmosphérique de PM mesurée dans les zones industrielles et aux abords des principaux axes routiers ont des taux supérieurs de $31 \%$ aux valeurs enrégistrées dans les banlieux. Plusieurs auteurs comme Cavanagh et al. (2009), Mitchell et Maher (2009), Dias et al. (2012), Barima et al. (2014) ont obtenus des résultats semblables. Aussi, le SIRM le plus élevé $\left(22,23 \times 10^{-5} \mathrm{~A}\right)$ obtenu avec les feuilles de $A$. spinosus est supérieur à celui obtenu par Barima et al. (2014), qui est de 4,51 x10${ }^{5} \mathrm{~A}$ avec la même espèce dans la même zone d'étude en début de saison sèche (novembre - décembre 2011). Ces différences peuvent s'expliquer par l'influence du microclimat entre les sites d'échantillonnage (Gromke et al., 2008 ; Mitchell et al., 2010 ; Górka-Kostrubiec et al., 2012). Les SIRM des feuilles obtenus en Côte d'Ivoire sont également supérieurs à la valeur maximale $(13,5$ $\mathrm{x} 10^{-5} \mathrm{~A}$ ) obtenue en milieu tempéré au mois de septembre à Gent ( Belgique) par Kardel et al. (2012). Selon Gromke et al. (2008), ces résultats entre les zones tempérée et 


\section{N'gouran et al. J. Appl. Biosci. 2014. Distribution spatiale intra-urbaine des particules fines : Monitoring}

par SIRM des feuilles en milieu urbain, Cote d'Ivoire

tropicale peuvent être dus par la différence de climats. Ce constat peut aussi s'expliquer par la source et le type de polluants (Gromke \& Ruck, 2009). Le taux de PM est élevé au niveau des principaux axes routiers à causes de l'émission des gaz d'échappement des nombreux véhicules (Moreno et al., 2003). A ces gaz d'échappement, l'on peut aussi ajouter les PM qui sont issues des produits d'abrasion de l'asphalte et du système de freinage des véhicules (Hoffmann et al., 1999). Dans les zones industrielles également, la valeur élevée de SIRM des feuilles enrégistrée est la conséquence du même phénomène de combustions incomplètes issues des cheminées des usines (Hunt et al., 1984 ; Flanders, 1994).

Différence entre les SIRM des feuilles suivant les espèces : L'analyse des résultats de cette campagne a montré une différence significative entre les SIRM des feuilles de $A$. spinosus et de $P$. maximum enrégistrés en mars ainsi qu'en juin (Tableau 1). Aussi, les feuilles de $A$. spinosus concernant les herbacées d'une part et celles de $F$. Benjamina et de $T$. catappa concernant les arborées d'autre part, ont des SIRM élevés dans cette étude. Cette grande valeur de SIRM enrégistrée au niveau de $A$. spinosus $\left(22,23 \times 10^{-5} \mathrm{~A}\right)$ exprime la capacité des feuilles de cette espèce à intercepter les PM. Cette capacité est due entre autre à la présence de poils sur les deux faces des feuilles de cette plante (Mitchell et al., 2010 ; Barima et al., 2014). En effet, les travaux de Mitchell et al. (2010) révèlent que les feuilles des plantes qui possèdent des poils sur leurs faces telles que Acer pseudoplatanus L. (Sapindaceae), Betula pendula Roth (Betulaceae), Fagus sylvatica L. (Fagaceae), Fraxinus excelsior L. (Oleaceae), Sambucus nigra L. (Adoxaceae) et Tilia platyphyllos Scop. (Tiliaceae) accumulent beaucoup plus de PM, comparées aux espèces qui ont des feuilles glabres dont Acer campestre $\mathrm{L}$. (Sapindaceae), Salix alba L. (Salicaceae) et Ulmus procera Salisb. (Ulmaceae) issues des mêmes milieux tempérés. Les résultats de cette étude ont montré également que les SIRM des feuilles des arborées ( $F$. benjamina et $T$. Catappa) sont en général plus élevés que les SIRM des herbacées exception faite pour le SIRM de $A$. spinosus $\left(22,23 \times 10^{-5} \mathrm{~A}\right)$ enregistré en zone industrielle. Plusieurs études ont montré que la grande surface foliaire des arbres (Sæbø et al., 2012), comme cela est le cas pour $T$. catappa, et la présence de cire sur les feuilles (Sæbø et al., 2012), comme pour F. benjamina, et la structure (tige, branches) des arborées sont des facteurs qui favorisent l'accumulation des PM par les arbres par rapport aux herbes (Fowler et al.,
1989). En plus, la faculté de production de latex par $F$. benjamina ferait de cette espèce une excellente bioindicatrice de la pollution en PM (Reyes et al., 2012). SIRM des feuilles aux abords des axes routiers : Le SIRM enrégistrés au niveau des feuilles de $A$. spinosus et de $P$. maximum diminuent progressivement lorsqu'on s'éloigne de l'axe routier (Figure 3). Ces résultats sont en accord avec ceux de plusieurs auteurs (Matzka \& Maher, 1999 ; Moreno et al., 2003 ; Kardel et al., 2012 ; Rooney et al., 2012; Barima et al., 2014). Ces auteurs ont indiqué que la principale source de pollution aux abords des routes est la production des gaz d'échappement. En effet, la concentration des principaux gaz primaires résultant de la circulation automobile $\left(\mathrm{CO}, \mathrm{NO}, \mathrm{NO}_{2}\right)$ est élevée dans le voisinage immédiat de la route puis diminue avec la distance et enfin ces gaz se diluent dans l'atmosphère (Kozawa et al., 2012 ; Baldauf et al., 2013).

Effet de la hauteur de prélevement des feuilles : Un gradient décroissant avec la hauteur de prélèvement est observé avec les SIRM des feuilles de $F$. benjamina et $T$. catappa (Fig. 4). En effet, les SIRM des feuilles des deux plantes sont plus élevés dans les premiers mètres ( 0 à 2 $\mathrm{m})$ au-dessus du sol qu'au delà confirmant que la source de pollution est la circulation automobile. Aux abords des routes, la pollution provient des produits d'abrasion de l'asphalte et des systèmes de freinage de véhicules (Hoffmann et al., 1999), mais aussi du type de carburant (diesel principalement) utilisé par ces véhicules (Sagnotti \& Winkler, 2012 ; Speak et al., 2012). En outre, Barima et al. (2014) ont constaté que la valeur du SIRM des feuilles de $F$. benjamina échantillonnées aux abords des routes a diminué de $62 \%$ au niveau de la canopée par rapport aux hauteurs inférieures proches donc du sol. Micallef et Colls (1998) ont aussi observé que les concentrations moyennes journalières et la fraction inhalable de PM dans une rue de la Grande Bretagne à $0,81 \mathrm{~m}$ de haut sont de $35 \%$ et de $12 \%$ supérieures par rapport à une hauteur supérieure de 2,88 m. Ces observations pourraient être expliquées au niveau des axes routiers par le poids et la taille des PM provenant des véhicules motorisés qui limitent leur accumulation en hauteur (Moreno et al., 2003). Concernant les zones industrielles, la faible concentration du SIRM des feuilles en hauteur bien que les PM soient émises à partir des cheminées des usines s'expliquerait plutôt par des causes exogènes telles que la pluie et le vent.

Influence de la saison sur le SIRM des feuilles : Le SIRM des feuilles des espèces étudiées est faible en saison pluvieuse par rapport à la saison sèche (Tableau1). La raison principale pourrait être le lessivage 
des feuilles par la pluie au cours de la saison pluvieuse. En effet, selon Matzka et Maher (1999), la pluie peut réduire à hauteur d'environ $80 \%$, les teneurs des SIRM des feuilles. Des tendances semblables ont été obtenues par Zhang et al., (2006) sur des feuilles de Pinus pumila

\section{CONCLUSION}

Cette étude a montré que les SIRM des feuilles de $A$. spinosus, $P$. maximum, $F$. benjamina et $T$. catappa en milieu tropical varient selon les espèces et le type d'utilisation du sol, mais aussi selon plusieurs autres facteurs tels que la distance de la plante par rapport à l'axe routier, la hauteur de prélèvement des feuilles et la saison d'échantillonnage. Les résultats de ces travaux ont révélé que les SIRM des feuilles sont plus élevés au niveau des axes routiers et des zones industrielles par rapport aux zones résidentielles et aux parcs et jardins. Ainsi les deux premiers types d'utilisation du sol peuvent être considérés comme étant des habitats de mauvaise qualité d'air contrairement aux zones résidentielles et aux parcs et jardins qui sont relativement de meilleure qualité d'air. En outre, l'effet saisonnier sur la concentration des particules fines des plantes tropicales est indéniable, car en saison de pluies, les

\section{REMERCIEMENTS}

Les auteurs remercient la Fondation David et alice Van Buuren et la Commission de Coopération au Développement (ex CIUF-CUD) pour avoir financé les travaux sur le terrain, et le VLIR UOS pour avoir permis l'analyse des échantillons en Belgique. Le troisième auteur est bénéficiaire du financement de Programme

\section{REFERENCES BIBLIOGRAPHIQUES}

Ahoussi KE, Loko S, Koffi YB, Soro G, Oga YMS, Soro $\mathrm{N}, 2013$. Evolution spatio-temporelle des teneurs en nitrates des eaux souterraines de la ville d'Abidjan (Côted'Ivoire). Int J Pure Appl Bios 3: 45-60.

Baldauf RW, Heist D, Isakov V, Perry S, Hagler GSW, Kimbrough S, Shores R, Black K, Brixey L, 2013. Air quality variability near a highway in a complex urban environment. Atmos Environ 64 : 169-78.

Barima YSS, Angaman DM, N'Gouran KP, Koffi NA, Kardel F, Cannière DC, Samson R, 2014. Assessing atmospheric particulate matter distribution based on Saturation Isothermal Remanent Magnetization of herbaceous and
Pall. (Pinaceae). Cependant, ce lessivage serait variable en fonction des caractères morphologiques des feuilles, de l'intensité de la pluie et de la position de la canopée (Mitchell et al., 2010).

SIRM des feuilles sont moins importants par rapport à la saison sèche. "Les différentes variations des SIRM des feuilles suivant la hauteur de prélèvement et la distance à la route ont confirmé que la source principale de pollution atmosphérique aux abords des principaux axes routiers est effectivement les gaz d'échappement issus des véhicules motorisés. L'ensemble des résultats obtenus dans cette étude montre que dans un environnement urbain, les plantes des zones tropicales pourraient être des bio-indicatrices de la pollution de l'air en particules fines à travers les SIRM des feuilles. Les pays en voie de développement pourraient donc trouver dans le biomonitoring une solution accessible à moindre coût pour estimer la qualité de l'air des villes. Ainsi, les décideurs publiques pourraient éloigner les populations des principales sources de pollution (axes routiers et zones industrielles) afin de préserver leur santé.

d'Appui Stratégique de l'Enseignement Supérieur. Enfin, nous sommes reconnaissants au Professeur Roeland Samson de l'University of Antwerp (Belgique) pour avoir accepté que nous analysions les échantillons dans son laboratoire.

tree leaves in a tropical urban environment. Sci Total Environ 470-471: 975-982.

Bealey WJ, McDonald AG, Nernitz E, Donovan R, Dragosits U, Duffy TR, Fowler D, 2007. Estimating the reduction of urban PM10 concentrations by trees within an environmental information system forplanners. J Environ Manage 85: 44-58.

Beaumont R, Hamilton RS, Machin N, Perks J, Williams ID, 1999. Social awareness of air quality information. Sci Total Environ 235: 319-329

Cavanagh JAE, Zawar-Reza P, Wilson JG, 2009. Spatial attenuation of ambient particulate matter air pollution within an urbanised native forest patch. Urban For Urban Gree 8: 21- 30. 
Dias D, Tchepel O, Carvalho A, Miranda Al, Borrego C, 2012. Particulate matter and health risk under a changing climate: assessment for Portugal. Sci World J 2012: 1-10.

Emberson LD, Ashmore MR, Murray F, Kuylenstierna JCl, Percy KE, Izuta T, Zheng Y, Shimizu H, Sheu BH, Liu CP, Agrawal M, Wahid A, AbdelLatif NM, Van Tienhoven M, De Bauer LI, Domingos M, 2001. Impacts of air pollutants on vegetation in developing countries. Water Air Soil Pollut 130: 107-118.

Flanders P.J, 1994.Collection, measurement, and analysis of airborne magnetic particulates from pollution in the environment, J Appl Phys 75: 5931-5936.

Fowler D, Cape JN, Unsworth MH, 1989.Deposition of atmospheric pollutants on forests. Phil Trans $R$ Soc Lond 324B: 247-65.

Georgeaud VM, Rochette P, Ambrosi JP, Vandamme D, Williamson D, 1997. Relationship between heavy metals and magnetic properties in a large polluted catchments, the Etang de Berre (South France).Phys Chem Earth 22: 211-214.

Górka-Kostrubiec B, Król Eb, Jeleńska M, 2012. Dependence of air pollution on meteorological conditions based on magnetic susceptibility measurements: a case study from Warsaw. Stud Geophys Geod 56: 861-77.

Gromke C, Ruck B, 2009. Effects of trees on the dilution of vehicle exhaust emissions in urban street canyons. Int J Environ Waste Manage 4: 22542.

Gromke C, Buccolieri R, Di Sabatino S, Ruck B, 2008. Dispersion modeling study in a street canyon with tree planting bymeans of wind tunnel and numerical investigations evaluation of CFD data with experimental data. Atmos Environ 42: 864050.

Hoffmann V, Knab M, Appel E, 1999. Magnetic susceptibility mapping of roadside pollution. J Geochem. Explor 66: 313-326.

Hunt A, Jones J, Oldfield F, 1984. Magnetic measurements and heavy metals in atmospheric particulates of anthropogenic origin, Sci Total Environ 33: 129-139.

INS, 1998. Recensement général de la population et de I'habitation, Côte d'Ivoire.

Kardel F, Wuyts K, Maher BA, Hansard R, Samson R, 2011. Leaf saturation isothermal remanent magnetization (SIRM) as a proxy for particulate matter monitoring: inter-species differences and in season variation. Atmos Environ 45: 5164-71.

Kardel F, Wuyts K, Maher BA, Samson R, Hansard R, 2012. Intra-urban spatial variation of magnetic particles: monitoring via leaf saturation isothermal remanent magnetisation (SIRM). Atmos Environ 55: 111-20.

Kozawa KH, Winer AM, Fruin SA, 2012. Ultrafine particle size distributions near freeways: effects of differing wind directions on exposure. Atmos Environ 63: 250-60.

Matzka J, Maher BA, 1999. Magnetic biomonitoring of roadside tree leaves: identification of spatial and temporal variations in vehicle-derived particulates. Atmos Environ 33: 4565-9.

Micallef A, Colls JJ, 1998. Variation in airborne particulate matter concentration over the first three metres from ground in a street canyon: implications for human exposure. Atmos Environ 32 : 3795-3799.

Mitchell R, Maher BA, 2009. Evaluation and application of biomagnetic monitoring of traffic- derived particulate pollution. Atmos Environ 43: 2095103.

Mitchell R, Maher BA, Kinnersley R, 2010. Rates of particulate pollution deposition on to leaf surfaces: temporal and inter-species magnetic analyses. Environ Pollut 158: 1472-8.

Moreno E, Sagnotti L, Winkler A, Dinares-Turell J, Cascella A, 2003. Biomonitoring of traffic air pollution in Rome using magnetic properties of tree leaves. Atmos Environ 37: 2967-77.

Nali C.,Lorenzini G, 2007. Air quality survey carried out by school children: An innovative tool for urban planning. Environ Monit Assess 131: 201- 210.

OMS, 2003. Health Aspects of Air Pollution with Particulate Matter, Ozone and Nitrogen Dioxide.World Health Organization, Bonn. Report on a WHO Working Group.

Prajapati SK, Pandey SK, Tripathi BD, 2005. Monitoring of vehicles derived particulates using magnetic properties of leaves. Environ Monit Assess 120: 169-175.

Reyes BA, Ruiz RC, Martínez-Cruz J, Bautista F, Goguitchaichvili A, Carvallo C, Morales J, 2012. Ficus benjamina leaves as indicator of atmospheric pollution: a reconaissance study. Stud Geophys Geod. 56: 879-887.

Rooney MS, Arku RE, Dionisio KL,Paciorek C,Friedman $A B$, Carmichael $H$, Zhou Z, Hughes AF, 
Vallarino J, Agyei-Mensah S, Spengler JD, Ezzati M, 2012. Spatial and temporal patterns of particulate matter sources and pollution in four communities in Accra, Ghana.Sci Total Environ 435-436: 107-14.

Sæbø A, Popek R, Nawrot B, Hanslin HM, Gawronska H, Gawronski SW, 2012. Plant species differences in particulate matter accumulation on leaf surfaces. Sci Total Environ 427- 428: 347-354.

Sagnotti L, Winkler A, 2012. On the magnetic characterization and quantification of the superparamagnetic fraction of traffic-related urban airborne PM in Rome, Italy. Atmos Environ 59: 131-40.

Speak AF, Rothwell JJ, Lindley SJ, Smith CL, 2012. Urban particulate pollution reduction by four species of green roof vegetation in a UK city.Atmos Environ 61: 283 - 93.

Zhang C, Huang B, Li Z, Liu H, 2006. Magnetic properties of high-road-side pine tree leaves in Beijing and their environmental significance. Chin Sci Bull 51: 3041-3052. 